http://dx.doi.org/10.12775/szhf.2017.018

ANDRZej LORENZ

Uniwersytet WrocŁawski, WrocŁaw, Polska

LORENZ@UNI.WROC.PL

\title{
Das Problem der „Dinge an sich selbst“ bei Kant im Lichte einer sprachanalytischen Rekonstruktion
}

\section{Einleitung}

Es gibt innerhalb der Transzendentalphilosophie Kants keine vergleichbare Begriffsbildung, die in der Kantforschung so umstritten und Auslöser so vieler Mißverständnisse wäre, wie das Theorem der „Dinge an sich selbst“. Diese Tatsache ist zum Teil damit zu erklären, daß Kant die theoriebildende Unterscheidung in „Erscheinungen“ und „Dinge an sich“ durch den vernunftkritischen Aufweis apriorischer Denk- und Anschauungsformen hinreichend vollzogen und zum Grundstein seiner epochemachenden Transzendentalphilosophie gemacht, diese Unterscheidung selbst aber in seinen transzendentallogischen Gedankengängen weder systematisch noch methodisch differenziert durchstrukturiert hat. Dadurch verstrickt er sich zwangsläufig in folgenschwere Ambiguitäten, die seiner Transzendentalphilosophie zunächst in der unmittelbaren Nachfolge ihrer deutschidealistischen Interpreten, anschließend lange Zeit innerhalb der Kant-Rezeption selbst zum Verhängnis 
einer schwerwiegenden Fehldeutung wurden, ohne daß die widerspruchsfreie Kohärenz seiner Erfahrungstheorie einstimmig konzediert wäre.

Kants erfahrungstheoretischem Transzendentalansatz zufolge käme es einer Ungereimtheit gleich, „Dinge an sich selbst“ erkennen zu wollen, d. h. wenn man von einem Gegenstand mehr erkennen wollte, als zu seiner möglichen Erfahrung gehört. Eine viel größere Ungereimtheit aber wäre, wenn man gar keine Dinge an sich annehmen würde (vgl. Prol., A 163), und das obwohl Kant ausdrücklich betont: „Was die Dinge an sich sein mögen, weiß ich nicht, und brauche es auch nicht zu wissen, weil mir doch niemals ein Ding anders, als in der Erscheinung vorkommen kann" (KrV B 333/334)1. Diese auf den ersten Blick paradoxe Verfahrenheit stellt jeden Interpreten auch heute noch vor ein offenes Problem, das bei seiner Lösung eine Reihe von Deutungsvorschlägen nahelegt, die deutlich voneinander divergieren.

Es ist anzunehmen, (1) daß das entwicklungsgeschichtlich mühsam erkämpfte Problem der Dinge an sich in seiner weiteren Durchführung für Kant selbst nicht mehr relevant war; (2) daß Kant, wie Schopenhauer vermutet, sich in puncto der Dinge an sich nicht mehr ganz klar geworden sei; oder (3) wie Gerold Prauss in seiner sprachanalytischen Untersuchung plausibel zeigt, in Kants Lehre vom Ding an sich liege eine infolge einer unklaren Begriffsformulierung entstandene Vermischung des transzendental-philosophischen mit dem transzendent-metaphysischen Charakter der Dinge an $s$ sich$^{2}$. Hätte Kant - so Prauss' Schlußfolgerung - den erfahrungstheoretischen Ansatz von vornherein konsequent durchgeführt, und daraufhin den genuin transzendental-philosophischen Sinn der „Dinge an sich selbst“ konzeptibel abgesteckt, so wäre der Unsinn ihrer transzendent-metaphysischen Fehldeutung bei seinen Nachfolgern sicherlich vermieden worden. So aber stellt das Problem der Dinge an sich eine der ungelösten und immer wieder kontrovers diskutierten Fragen der Kantrezeption dar, an der sich die Geister bis heute trennen.

\footnotetext{
${ }^{1}$ Kants Werke werden zitiert nach der Ausgabe: Immanuel Kant. Werkausgabe in 12 Bänden, Hrsg. v. Wilhelm Weischedel. Frankfurt/M. 1974. Dabei werden folgende Sigeln verwendet: Kritik der reinen Vernunft $(K r V)$; Prolegomena zu einer jeden künftigen Metaphysik, die als Wissenschaft wird auftreten können (Prol.); Über die von der Köngl. Akademie der Wissenschaften zu Berlin für das Jahr 1791 ausgesetzte Preisfrage: Welches sind die wirklichen Fortschritte, die die Metaphysik seit Leibnizens und Wolffs Zeiten in Deutschland gemacht hat? (FdM); Grundlegung zur Metaphysik der Sitten (GMS).

${ }^{2}$ G. Prauss, Kant und das Problem der Dinge an sich, Bonn 1974, S. 9; ders.: Erscheinung bei Kant. Ein Problem der „Kritik der reinen Vernunft“, Berlin 1973, S. 15 ff.
} 
Der folgende Rekonstruktionsversuch stellt sich erneut dem Problem der Dinge an sich bei Kant ${ }^{3}$. Dabei wird strikt unterschieden zwischen Kants eigener Konzeption der Dinge an sich selbst, die ihrem Ansatz nach als kohärent betrachtet wird, und ihrer Fehldeutung bei seinen Nachfolgern. Das Hautgewicht wird dabei auf die sprachanalytische Freilegung der ursprünglichen Bedeutung des vollständigen Ausdrucks „Dinge an sich selbst betrachtet“als originärem Sinnverständnis von Kants Lehre gelegt.

\section{Der transzendentalphilosophische Sinn der Unterscheidung „Ding als Erscheinung“ und „Ding an sich selbst“ betrachtet}

Der vollständige Ausdruck „Dinge an sich selbst“ kann innerhalb der Transzendentalphilosophie Kants in zweierlei Hinsicht verstanden werden: (1) in einem transzendental-philosophischen Sinne, auf den Kant mit seiner metaphysischen Erfahrungstheorie eigentlich hinaus wollte, und der sich in seiner Philosophie als mit zureichenden Argumentationsmitteln begründet erweist; und (2) in einem transzendent-metaphysischen Sinne, der von den Kritikern immer wieder ins Treffen geführt wird, der bei genauerer Analyse aber sich als unhaltbar erweist. Er tritt immer wieder dann zutage, wenn Kant bei der methodologischen Durchführung seinen ursprünglichen Denk- und Erfahrungsansatz nicht hinreichend im Sinne von „Ding als Erscheinung“ und „Ding an sich selbst betrachtet" entfaltet ${ }^{4}$.

Ein entscheidender Grund für diese Inkonsequenz liegt sicherlich in Kants entwicklungsgeschichtlicher Variabilität selbst. Denn bevor Kant das „Ding an sich selbst" in der endgültigen Form seiner erfahrungstheoretischen Unerkennbarkeit in der Kritik der reinen Vernunft (1781) eingeführt hatte, vertrat er noch in der Dissertation De mundi sensibilis atque intelligibilis forma et principiis (1770) die Auffassung, daß die Sinne die Dinge so darstellen, wie sie

\footnotetext{
${ }^{3}$ Vgl. dazu A. Lorenz, Das Problem der Dinge an sich bei Kant, Schopenhauer und Freud, Wrocław 2004. Ferner vgl. A. Lorenz, „Problem rzeczy samych $w$ sobie” $w$ filozofii transcendentalnej Kanta - próba rekonstrukcji na podstawie analizy językowej, in: Idea transcendentalizmu. Od Kanta do Wittgensteina, P. Parszutowicz/M. Soin (red.), Warszawa 2011, S. 51-76.

${ }^{4}$ G. Prauss, Kant und das Problem der Dinge an sich, S. 9 f. Vgl. J. Mittelstraß, Ding als Erscheinung und Ding an sich. Zur Kritik einer spekulativen Unterscheidung, in: J. Mittelstraß und M. Riedel (Hg), Vernünftiges Denken. Studien zur praktischen Philosophie und Wissenschaftstheorie, Berlin/New York 1978, S. 116 f.
} 
„erscheinen“ (uti apparent); der Verstand sie dagegen in einer Weise darstelle, wie sie „an sich“ sind (sicuti sunt): „Sensitive cogitata esse rerum repraesentationes uti apparent, intellectualia autem sicuti sunt“ (A 8). Zu berücksichtigen dabei ist, daß Kant vom Standpunkt der Dissertation aus noch lediglich zwischen einem "realen“ und einem „logischen“ Verstandesgebrauch unterschieden hat. Während der „logische Gebrauch“ der Verstandesbegriffe allein für die Sinnlichkeitserkenntnis als Erkenntnis von Erscheinungen zu gelten hatte, sollte der "reale Gebrauch“ die Dinge an sich als Noumena erkennen können.

Mit der Kopernikanischen Umänderung der Denkungsart der Vernunftkritik aber wird der „logische“ Gebrauch zum „transzendentalen“ reformuliert. Nun heißt es zwar weiterhin, „die Sinne stellen uns die Gegenstände vor, wie sie erscheinen, der Verstand aber, wie sie sind“, dennoch ,ist das letztere nicht in transzendentaler, sondern bloß empirischer Bedeutung zu nehmen, nämlich wie sie als Gegenstände der Erfahrung, im durchgängigen Zusammenhange der Erscheinungen, müssen vorgestellt werden, und nicht nach dem, was sie, außer der Beziehung auf mögliche Erfahrung, und folglich auf Sinne überhaupt, mithin als Gegenstände des reinen Verstandes sein mögen. Denn dieses wird uns immer unbekannt bleiben, so gar, daß es auch unbekannt bleibt, ob eine solche transzendentale (außerordentliche) Erkenntnis überall möglich sei, zum wenigsten als eine solche, die unter unseren gewöhnlichen Kategorien steht. Verstand und Sinnlichkeit können bei uns nur in Verbindung Gegenstände bestimmen“ (KrV B 313/314).

Transzendental bezeichnet von jetzt an nur die Erkenntnismöglichkeit der Anwendung des Apriorischen auf die Erfahrung und seine Gegenstandsgeltung. Transzendental ist alles, was in bezug auf Bedingungen möglicher Erfahrung als gegenstandskonstituierende Denkvoraussetzungen gilt und bedeutet "niemals eine Beziehung unserer Erkenntnis auf Dinge, sondern nur aufs Erkenntnisvermögen" (Prol., A 71). Transzendental heißt somit alle Erkenntnis, „die sich nicht so wohl mit Gegenständen, sondern mit unserer Erkenntnisart von Gegenständen, so fern diese a priori möglich sein soll, überhaupt beschäftigt" ( $K r V$ B 25). Darüber hinaus impliziert die vernunftkritische Unterscheidung in eine Sinnen- und eine Verstandeswelt als Differenzierung zwischen Phaenomena und Noumena moralphilosophische Begründungsziele des transzendentalen Handlungssubjekts als Ding an sich, um den intelligiblen Freiheitscharakter des Menschen (als Vernunftwesen) überhaupt rechtmäßig designieren zu können ${ }^{5}$.

${ }^{5}$ Kants praktische Philosophie als Metaphysik der Sitten, in der der Mensch als moralisches Handlungssubjekt mit dem „Ding an sich“ ineinsgesetzt wird, kann im Rahmen dieser 
Von seiner Kopernikanischen Drehwende wohl noch nichts ahnend, erklärt Kant in der Dissertation aufgrund der Sonderung von Sinnes- und Verstandeserkenntnis die „Dinge an sich“ als Noumena in Abhebung von aller Sinnlichkeit mittelst reiner Verstandesbegriffe zunächst noch für erkennbar. Das transzendentale Problem der Dinge an sich ist in seiner entwicklungsgeschichtlichen Segmentierung mit einem dogmatischen Begriffsrest insofern belastet, als Kant bei seiner vernunftkritischen Lösung auf konzeptionelle Vorleistungen aus der vorkritischen Phase zurückgreift und sie in eine erkenntnistheoretisch diametral veränderte Problemlösung einzuschließen sucht. Die vernunftkritische Reflexionswandlung verwirft nämlich radikal die Annahme der erfahrungstheoretischen Erkennbarkeit der Dinge an sich.

Gerold Prauss hat gezeigt, daß das Problem der Dinge an sich bei Kant mit den Mitteln der sprachanalytischen Philosophie dennoch plausibel zu lösen ist. Es kann aus dem Textlaut der Vernunftkritik analytisch herausgelöst und vom Standpunkt der Sprachanalyse aus im Rahmen einer Theorie zweistufiger Reflexion systematisch rekonstruiert werden, sofern die Grundterme „Erscheinung" und „Ding an sich“ als nichtempirische Denkkorrelate transzendentaler Reflexion zweiter Stufe nicht auf irgendwelche transzendenten "Dinge-an-sich“ angewandt, sondern im Anschluß an den auf der ersten Stufe freigelegten erfahrungstheoretischen Sinn ausschließlich in bezug auf das Empirische gebracht werden. Auffallenderweise aber wird das Theorem der „Dinge an sich“ in der einschlägigen Kantliteratur von Anfang seiner Rezeptionsgeschichte an ,auf den transzendent-metaphysischen Unsinn des Ausdrucks, Dinge an sich' festgelegt" ${ }^{\text {"6 }}$.

Der erfahrungstheoretische Ansatz aber - auf den Kant ursprünglich mit der transzendentalen Empirienähe der „Dinge an sich“ eigentlich abgesehen hat - geht dabei unversehens verloren. Daraus entspringt ein fundamentales Mißverständnis, das in seiner weiteren Rezeptionsgeschichte nicht auf den methodischen Grundcharakter von transzendentalen Objekten, sondern gerade auf den metaphysischen Unsinn von transzendenten Entitäten theoriekonträr festgelegt wird. Für Kant selbst aber war das Theorem der „Dinge an sich selbst (und nicht als Erscheinungen) betrachtet" kein fauler Fleck einer

Untersuchung weder berücksichtigt noch behandelt werden. Allein der transzendentalphilosophische Erfahrungscharakter der Dinge an sich kann hier berücksichtigt werden, und zwar ganz im Sinne Kants: „Daher ist die Transzendental-Philosophie eine Weltweisheit der reinen bloß spekulativen Vernunft. Denn alles Praktische, so fern es Triebfedern enthält, bezieht sich auf Gefühle, welche zu empirischen Erkenntnisquellen gehören“" $K r V$ B 29).

${ }^{6}$ G. Prauss, Kant und das Problem der Dinge an sich, S. 10. 
metaphysischen Hinterwelt, wie dies geflissentlich Nietzsche zu quittieren meinte, sondern ein im Kontext der Unterscheidung aller Gegenstände in Phänomena und Noumena rein methodischer Ausdruck, es war - um Kant gegen jeden Mißbrauch selbst zu Wort kommen zu lassen - „ein Grenzbegriff, um die Anmaßung der Sinnlichkeit einzuschränken, und also nur von negativem Gebrauche" (KrV B 311/312).

Eher wir uns der Problemfrage selbst zuwenden, ist zunächst zu klären, welche satzsystematische Strukturfunktion der Gesamtausdruck „Dinge an sich selbst" bei Kant denn eigentlich aufweist. Im folgenden wird im Anschluß an Gerold Prauss von der Annahme ausgegangen, daß die Rede von Dingen an sich im transzendental ursprünglichen Sinne der Kantischen Erfahrungstheorie die abschließende Grundfunktion erfüllt, diese Theorie als eine nichtempirische Theorie des Empirischen zu vollenden. Ihr transzendental-philosophischer Sinn im Gegensatz zu dem metaphysisch-transzendenten Unsinn besteht demnach darin, daß die Transzendentalphilosophie neben Mathematik und Physik als synthetisch-apriorische Vernunftwissenschaft von eigener Art auf dem Wege transzendentalkritischer Reflexion auf das Empirische führt, das in rationaler Argumentation die Einsicht in eine nichtempirische Theoriedimension dieses Empirischen freilegt.

Von „Dingen an sich“ läßt sich im Rahmen dieser Metatheorie erst dann sinnvoll reden, wenn man die Gegenstände als Objekte sinnlicher Erfahrung zu subjektabhängigen Erscheinungen erklärt. Zum einen stellt das Ding an sich seiner Denkbarkeit nach das transzendental differenzierte Korrelat zu Erscheinung dar. Zum anderen sind die „Dinge an sich selbst“ ein durchweg negativer Ausdruck, der aus seiner Negation in bezug auf den Gegenausdruck „Erscheinungen“ resultiert. Das „Ding an sich“ ist seinen exemplarischen Unterscheidungsmerkmalen nach anderen „Dingen an sich“ gegenüber nicht differenzierbar. Die konzeptionelle Korrelation Erscheinung und Ding an sich selbst hat es aber an sich, daß es notwendig ist, die Dinge nicht nur als „Erscheinungen“, sondern auch „an sich selbst“ zu betrachten, was wiederum heißt, sie gerade nicht als Erscheinungen zu denken. Erst diese doppelte, dem erfahrungstheoretischen Transzendentalansatz wesentliche Betrachtungsweise macht es sinnvoll, in gänzlicher Absetzung von Erscheinungen auch von der Betrachtung der „Dinge an sich selbst“ im transzendentalen Sinne zu sprechen. „An sich selbst“ heißt demnach nichts anderes als „nicht als Erscheinung“.

Das Ergebnis der sprachanalytischen Rekonstruktion des Reflexionsausdrucks „Dinge an sich“ legt den Schluß nahe, daß der Standardausdruck 
„Ding an sich selbst“ auf die eigentlich philosophische Betrachtung nicht im Sinne einer metaphysisch-transzendenten Erschließung von besonderen „Dingen-an-sich" hinleitet, sondern aufgrund der Differenzierung in empirische Erscheinungen und empirische Dinge an sich den Bereich des Erfahrbaren von einem transzendentallogischen als nichtempirischen Standpunkt aus durch den Grenzbegriff Ding an sich selbst nochmals abgrenzt, um gerade die transzendente Anmaßung der Sinnlichkeit in bezug auf eine vermeintliche Erkenntnis der „Dinge an sich“ einzuschränken.

Wenn man nämlich wie Kant sowohl den Standpunkt des transzendentalen Idealismus als auch den des empirischen Realismus teilt, so können aufgrund der Erklärung aller Gegenstände zu Erscheinungen diese Gegenstände als empirische Realität einmal als „Erscheinung“ und einmal als „Ding an sich selbst“ betrachtet werden. Dann aber bedeutet nach Kant „die Unterscheidung des so genannten Gegenstandes an sich im Gegensatz mit dem in der Erscheinung (phaenomenon adversus noumenon) nicht ein wirkliches Ding, was dem Sinnengegenstande gegenüber steht, sondern als = X nur das Princip, daß es nichts Empirisches sey, was den Bestimmungsgrund der Möglichkeit der Erfahrung enthält" (Op. post. II, 24) ${ }^{7}$. Der besondere transzendentale Sinn des Ausdrucks „Ding an sich selbst“ schöpft seine Grundbedeutung aus seiner sprachlichen Signifikanz. M. a. W., der eigentliche Sinnträger „an sich selbst" ist im Rahmen der transzendentalphilosophischen Reflexion - die ihrerseits auf den empirischen Zusammenhang zwischen Erscheinungen und Dingen an sich rekurriert - die adverbiale Bestimmung zu „betrachtet“.

Prauss folgert daraus, daß wenn Kant von „Dingen an sich selbst“ spricht, so spricht er stets von Dingen, auf die er in einer besonderen Weise nicht etwa durch adnominale Bestimmung zu Ding im Sinne von „Ding-an-sich“, sondern durch die adverbiale Bestimmung zu „betrachtet", und zwar „Ding, - an sich selbst betrachtet,“ transzendentalphilosophisch reflektiert. In diesem Sinne ist die folgenschwere Reduktion der Langform „Ding an sich selbst“ auf die Kurzform „Ding an sich“ im Sprachgebrauch der Kantliteratur als eine sinnentstellende Mißdeutung Kantischer Absichten, als Hypostasierung seines ursprünglichen Reflexionsausdrucks, abzulehnen. Der Ausdruck „Ding(e) an sich selbst" bringt nämlich die Bezugnahme auf die Betrachtungsart und nicht auf irgendwelche transzendenten „Dinge-an-sich" ${ }^{\text {“8 }}$.

\footnotetext{
${ }^{7}$ Opus postumum. Hrsg. v. Artur Buchenau, Berlin/Leipzig 1938.

${ }^{8}$ G. Prauss, Kant und das Problem der Dinge an sich, 23 u. 29. Wie Prauss anhand der Sprachanalyse gezeigt hat, verwendet Kant in mehr als 94\% aller Fälle die Langform „Dinge an
} 
Unter dieser semantisch differenzierten Einschränkung läßt sich dann lediglich die reflexionsimmanente Bezugnahme auf die Betrachtungsart selbst, wohlgemerkt aber nicht auf ein transzendentes Seiendes im Sinne eines Quasi-Eigennamens „Ding-an-sich“ erschließen. Die gängige Annahme der herkömmlichen Kantliteratur, Ausdrücke wie „Ding an sich selbst“ oder „Objekt an sich selbst" würden eine semantische und darüber hinaus eine metaphysische Einheit in einer Weise nahe legen, als ob Kant mit dieser Wendung eine unmittelbar einsehbare Bestimmung eines übersinnlichen „Dingansichseins" vornehmen würde, ist vom Standpunkt der Sprachanalyse als falsch zurückzuweisen. Festzuhalten demnach ist, daß Formulierungen in der Art von „Dingen-an-sich“ ominöse Hypostasierungen des besonderen Reflexionsausdrucks auf das Empirische bei Kant darstellen.

\section{Die empirische Differenz zwischen Erscheinung und Ding an sich}

Aufgrund der textanalytischen Unterscheidung zwischen dem empirischen und dem transzendentalen Sinncharakter des Begriffspaares "Erscheinung" und „Ding an sich selbst“ ist davon auszugehen, daß Dinge als Gegenstände, die zunächst als Erscheinungen betrachtet werden müssen, „an sich selbst“ zu betrachten, heißt, sie als Nicht-Erscheinungen nach der formalsynthetischen Kategorieneinheit nur zu denken. Daraus resultieren zwei unterschiedliche Betrachtungsweisen. Erstens, ihre aufgrund des empirischen Anschauungscharakters ausgewiesene Erkennbarkeit als empirische Erscheinungen in ihrem sinnlich wahrnehmbaren Empfindungsgehalt. Zweitens, ihre transzendentale Denkbarkeit, die ihre erfahrbare Erkennbarkeit in der Betrachtung als „Dinge an sich selbst“ ausschließt.

Erkenntnis entstammt nach Kant aus zwei Grundquellen, der Rezeptivität der Eindrücke, durch die ein zu erkennender Gegenstand zunächst empirisch gegeben, und der Spontaneität der Begriffe, durch die dieser Gegenstand in Relation auf eine solche Vorstellung gedacht wird (vgl. KrV B 74). Sinnliche Anschauungen und diskursive Begriffe stellen die Grundelemente jedweder

sich selbst“ oder entsprechend „Gegenstand“ bzw. „Objekt an sich selbst“, aber nur in 6\% die Kurzform „an sich“, die gerade in der Kant-Interpretation zum Standardausdruck geworden ist. Ibid., 28 u. $13 \mathrm{ff}$. 
Erkenntnisfähigkeit dar und bedingen sich in ihrem wechselseitigen Denkund Anschauungsverhältnis. Weder reine Begriffe ohne eine ihnen korrespondierende Anschauung noch Anschauungen ohne einen entsprechenden Verstandesbegriff können zu einer effektiven Gegenstandserkenntnis führen, oder - wie Kant selber sagt: „Gedanken ohne Inhalt sind leer, Anschauungen ohne Begriffe sind blind“ (KrV B 75).

Kant hat den Unterscheidungszusammenhang zwischen beiden Betrachtungsweisen und ihren Korrelata „Erscheinung“ und „Ding an sich“ in unterschiedlichem Sinne gebraucht; er hat ihn aber nur selten angemessen kenntlich gemacht. Daraus ist auf eine folgenschwere Vermischung der Betrachtungsebenen zu schließen, die dann vorliegt, wenn der transzendentale Sinn auch in einer primär empirischen Hinsicht gebraucht wird. Dabei tritt der Mangel einer konsequent durchgeführten metatheoretischen Differenzierung zwischen dem rein empirischen und dem transzendentalen Sinnträger des Begriffspaares „Escheinung“ und „Ding an sich“ offen zutage. Prauss zufolge bleiben explizite Unterscheidungen dieser Art bei Kant sogar seltene Ausnahmen: „In der Regel verwendet er vielmehr dieselben Ausdrücke ,Erscheinung' und ,Ding an sich' sowohl in ihrer empirischen als auch in ihrer philosophischen Bedeutung. Ja nicht nur das: Er wechselt nach Belieben, oft sogar im selben Satz, von der einen zur andern Bedeutung hinüber und läßt dabei sogar [...] diese so unterschiedlichen Bedeutungen oft genug ineinander fließen, so daß ihm dieser Unterschied und damit auch der besondere Sinn von ,Erscheinung ' und ,Ding an sich verloren zu gehen droht" ${ }^{\text {“9. }}$

Die Signifikanz dieser Unterscheidung besteht zunächst in einer semantischen Differenzierung zwischen „Erscheinung“ im empirischen und „Phaenomenon" im transzendentalen Sinne. Sofern Kant auf der empirischen Betrachtungsebene von empirischen Gegenständen als vorgestellten Objekten spricht, ist zwischen zweierlei empirisch Seiendem zu unterscheiden. Einem real gegebenen Ding, z. B. einer Rose, und einer Empfindung ggf. einer empirischen Sinnesanschauung, unter Vermittlung deren sie in einem objektiven Gegenstandsbezug überhaupt erst empirisch gegeben wird. Das empirisch Seiende präsentiert sich stets unter subjektiven Formstrukturen als „Erscheinung“ eines empirischen „Dinges an sich“. Es erscheint unter variierenden subjektiven Bedingungen der Sinnlichkeit und wird dem Erkennenden nie in seinem vorstellungsabgewandten Ansichsein, sondern in seiner Erscheinung gegeben, die je nach Verschiedenheit des subjektiven Standpunktes auch ver-

\footnotetext{
${ }^{9}$ Ibid., S. 52.
} 
schieden erscheinen kann: „Da nämlich etwa Farben, Geschmack etc. mit Recht nicht als Beschaffenheiten der Dinge, sondern bloß als Veränderungen unseres Subjekts, die so gar bei verschiedenen Menschen verschieden sein können, betrachtet werden. Denn in diesem Falle gilt das, was ursprünglich selbst nur Erscheinung ist, z. B. eine Rose, im empirischen Verstande für ein Ding an sich selbst, welches doch jedem Auge in Ansehung der Farbe anders erscheinen kann" ( $K r V$ B 45).

Von einem empirischen Standpunkt aus „Ding an sich selbst“ bedeutet ein „Noumenon im negativen Verstande“, das zwar transzendental denkbar, aber nicht sinnlich anschaubar und damit auch nicht objektiv vorstellbar ist: „Wenn wir unter Noumenon ein Ding verstehen, so fern es nicht Objekt unserer sinnlichen Anschauung ist, indem wir von unsrer Anschauungsart abstrahieren: so ist dieses ein Noumenon im negativen Verstande. Verstehen wir aber darunter ein Objekt einer nichtsinnlichen Anschauung, so nehmen wir eine besondere Anschauungsart an, nämlich die intellektuelle, die aber nicht die unsrige ist“ ( $K r V$ B 307). Der Ausdruck „Erscheinung“ kann sinngemäß im empirischen Sinne einer psychischen Empfindung gedeutet werden. Der empirische Sinn der Betrachtung als Erscheinung selbst besagt, daß hier Etwas von etwas gegensätzlich Anderem erscheint, das gerade nicht als Erscheinung betrachtet wird, und dieses erfahrungsmäßig unhintergehbare "Etwas" in seiner je eigenen Distinktion des empirisch Seienden als in seinem selbsteigenen „Ansich“ bewahrt bleibt.

Daraus wird ersichtlich, daß etwas mir oder jemand anderem erscheinen muß, und daß die Erscheinung eines „Ansich“ stets durch das Psychische der subjektiven Sinnesempfindung vermittelt wird. Alles über die Empfindung vermittelte Physische (Nicht-Subjektive) wird dennoch nicht als rein Psychisches (Subjektives) verstanden. Denn - wie Prauss einwendet - „obwohl es uns immer wieder nur durch das Subjektive unserer Empfindung oder Wahrnehmung erscheint, wird alles Objektive in der Erfahrung von uns doch gerade nicht als Subjektives betrachtet ${ }^{\text {"10 }}$. Daher erscheint es notwendig, wenn nicht gar unerläßlich, konsequent zwischen einer empirischen Erscheinung als einem Subjektiven und einem empirischen Ding an sich als einem Objektiven, von aller Erscheinung völlig Anderem als Gegensätzen, zu differenzieren. Der Interpretationslösung von Prauss folgend können wir insofern auch von „subjektiv-psychischen“ Erscheinungen und „objektiv-physischen“ Dingen sprechen.

\footnotetext{
${ }^{10}$ Ibid., S. 47.
} 
Eine empirische Erscheinung wird dennoch stets in ihrer intrasubjektiven Dependenz gegeben. Ein empirisches Ding an sich dagegen kann als intersubjektiver Gegenstand zum Objekt für jedes Subjekt werden. Als objektivphysisches Ding stellt es einen Gegenstand für mögliche Erfahrungserkenntnis dar, und weist auf die ontologische Distinktion des empirisch Seienden in seinem selbsteigenen Ansich hin. In seiner empfindungsmäßigen Erscheinung wiederum wird es von seinem Träger, dem sie wahrnehmenden Erkenntnissubjekt, abhängig. Mit dieser Unterscheidung liegt uns die empirische Betrachtungsebene als Differenz zwischen „Erscheinung“ und „Ding an sich“ eines im zweifachen Sinne empirisch Seienden rekonstruiert vor.

\section{Die transzendentale Differenz zwischen Erscheinung und Ding an sich}

Der Übergang von der empirischen zur transzendentalen Differenz zieht eine einschneidende Betrachtungsänderung nach sich. Denn Erscheinung im transzendentalen Sinne, „da man von Dingen sagt, sie sind Erscheinungen (phaenomena), ein Begriff von ganz anderer Bedeutung ist, als wenn ich sage, dieses Ding erscheint mir so oder so, welches die physische Erscheinung anzeigen soll“ (FdM A 33). Die explizite Differenzierung zwischen „Erscheinung“ und „Phaenomenon“ einerseits, „Ding an sich“ und „Noumenon“ andererseits, weist darauf hin, daß Kant den Unterschied zwischen dem empirischen und dem transzendentalen Sinn beider Grundterme gesehen und ihn auszuräumen gesucht hat. In bezug auf die Noumena spricht er sogar von einem erkenntnistheoretischen Phänomen, das er mit dem Namen „transzendentale Amphibolie" als Verwechslung des reinen Verstandesobjekts mit der Erscheinung bezeichnet (vgl. KrV B 325).

Als Transzendentalphilosoph stand Kant vor dem Problem einer Abgrenzung zwei konkurrierenden Erkenntnistheorien gegenüber. Zum einen mußte er seine Konzeption der Intellektualisierung Leibniz', zum anderen der Sensualisierung Lockes gegenüberstellen. Leibniz intellektualisierte die Erscheinungen, weil ihm, so Kant, die Erscheinung schon Vorstellung des Dinges an sich selbst war; Locke dagegen sensualisierte sie einseitig, indem er sie für rein empirische Begriffsbildungen hielt (vgl. $K r V$ B 327).

Für Kant selbst waren Verstand und Sinnlichkeit zwei unterschiedliche Erkenntnisquellen, die nur in einer Korrelationsverbindung objektive Gül- 
tigkeit der Erfahrungserkenntnis beanspruchen konnten, sofern synthetische Erkenntnis a priori begründbar sein sollte. Keine dieser Eigenschaften sollte der anderen vorgezogen werden, da Erfahrungsgegenstände nur unter Vermittlung von Kategorien denkbar sind. Kategorien als reine Verstandesformen aber stellen keine besonderen Objekte dar, sondern dienen lediglich dazu, das gedachte transzendentale X-Objekt durch das, was in der Sinnlichkeit gegeben wird, zu bestimmen, um Erscheinungen unter Gegenstandsbegriffe subsumieren zu können. Es ist die Aufgabe der transzendentalen Reflexion, die Begriffe unter Sinnlichkeitsbedingungen in einem Schematisierungsvorgang zu verbinden. Der Kategoriengebrauch selbst wiederum ist nur schematisiert unter Raumzeitbedingungen möglich, und bleibt stets auf Erscheinungen als Erfahrungsgegenstände restringiert.

Erscheinungen, sofern sie als Gegenstände nach der Einheit der Kategorien gedacht werden, heißen in Kants Sprachgebrauch Phaenomena. Damit setzt die Unterscheidung ,aller Gegenstände überhaupt in Phaenomena und Noumena" ein, denn im transzendentalen Reflexionssinn treten Erscheinung und Ding an sich selbst als Phaenomenon und Noumenon auf (vgl. KrV B 295 ff.). Während auf der Ebene des empirischen Dinges an sich - das an die Ergebnisse der Transzendentalen Ästhetik, die reinen Anschauungsformen Raum und Zeit, gebunden bleibt - Erscheinung und Ding an sich völlig different, ja geradezu divers waren, wird mit dem Übergang zum transzendentalen Sinn von Erscheinung und Ding an sich - der seinerseits an die Transzendentale Analytik, die reinen Verstandesbegriffe als Bedingungen der Denkbarkeit des Gegenständlichen, anknüpft - nicht mehr der empirische Gegenstand eines „Dinges an sich" in seinem Erscheinungscharakter betrachtet, sondern allein die spezifisch transzendentale Betrachtungsart selbst, die den auf der empirischen Betrachtungsebene ausgewiesenen Gegensatz zwischen empirischer Erscheinung und empirischem Ding an sich nun gerade als „,identisch“ setzt.

Auf der Ebene der Erfahrungserkenntnis stehen sich „Erscheinung“ und „Ding an sich“ gegensätzlich als Grundverschiedenes gegenüber, und zwar als das empirisch Seiende des subjektiv Psychischen und das empirisch Seiende des objektiv Physischen. Auf der transzendentalen Betrachtungsebene aber geht diese numerisch-existenzielle Differenz verloren. Dagegen wird eine numerisch-existenzielle Identität zwischen Erscheinungen und Dingen an sich virulent. Die besondere Schwierigkeit der eingeleiteten Betrachtungsänderung hängt mit dem Umstand zusammen, daß Kant die Reflexion auf die Betrachtbarkeit einmal „als Erscheinung“ und dann wiederum „nicht als Erscheinung", d. h. „, an sich selbst“, nicht nur auf das Physische, sondern auch 
auf das Psychische - das Subjekt selbst - überträgt ${ }^{11}$. Wenn aber im empirischen Sinne das objektiv Physische und das subjektiv Psychische aufgrund der Dualität des empirisch Seienden als je gegensätzlich aufzufassen waren, ändert sich im transzendentalen Sinne diese Betrachtungsweise grundlegend.

Die transzendentale Differenz schöpft ihren Gegensatz aus der Reflexionsschwierigkeit her, daß nun ohne jede empirische Bezugnahme etwas als Erscheinung und dann wiederum nicht als Erscheinung, d. h. an sich selbst, betrachtet wird, und was als signifikanter Gegensatz nicht aus dem Betrachteten selbst resultiert. Denn das Gegensätzliche wird aus der spezifisch transzendentalen Betrachtungsart selbst, und nicht wie bei der empirischen Betrachtung des empirischen Gegensatzes aus der Distinktion zwischen dem Psychischen und dem Physischen abgeleitet. Das Gegensätzliche bezeichnet nun nichts anderes als die transzendentale Reflexionsart selbst, zu deren Besonderheit es eben - mit Prauss gesprochen - gehört, „daß sie in so gegensätzlicher Weise jeweils ein und dasselbe ${ }^{\text {"12 }}$ aufgrund der kategorialen Denkbarkeit betrachtet, obwohl auf dieser Betrachtungsebene keine empirisch differenzierbare Anschauung mehr zugrunde liegt. Erst unter dieser Betrachtung erhält dieses Selbige bzw. Identische einen je gegensätzlichen Sinn.

Folgerichtig können wir festhalten, daß die empirische Betrachtungsweise der empirischen Dinge in ihrem Unterschied, sie nicht als Erscheinungen zu betrachten, keinerlei Gemeinsamkeit mit der rein transzendentalen Betrachtungsart hat. Beide sind sich gegenüber völlig indifferent, für den erfahrungstheoretischen Möglichkeitsaufweis synthetischer Erkenntnis a priori aber unabdingbar.

\section{Das Problem der Affektion durch Dinge an sich}

Neben dem Problem einer adäquaten Differenzierung zwischen Erscheinungen und Dingen an sich stellt sich eine weitere Schwierigkeit. Es ist die nicht weniger problematische Frage der Affektion als systematisches Hauptproblem der Kantischen Lehre von Dingen an sich. Wie ist der Vorgang zu erklären, daß durch äußere Gegenstandseinwirkung in einem mit sinnlichen Rezeptoren versehenen Affektionssubjekt eine signifikante Empfindung

\footnotetext{
${ }^{11}$ Ibid., S. 52.

${ }^{12}$ Ibid., S. 53.
} 
hervorgerufen wird und wie verhält sich hierzu der Prozeß begrifflicher Erkenntnisbildung. M. a. W., wie verhalten sich äußere und innere Erfahrung zueinander und was versteht Kant denn eigentlich unter Affektion? Relevant in diesem Zusammenhang ist nur der Affektionsweg, nicht der Vorgang selbst, der bei einer detaillierten Behandlung genügend andere Schwierigkeiten aufwerfen dürfte.

Kant zufolge kann ein Erfahrungsgegenstand dem Erkenntnissubjekt nur dadurch gegeben werden, daß er "das Gemüt auf gewisse Weise affiziere“, und dadurch Eindrücke als Sinnesempfindungen erzeugt. Objektive Dinge als empirisch gegebene Erfahrungsgegenstände affizieren die Sinne und rufen subjektive, ihrer Intensität nach variierende Sinnesempfindungen hervor. Auf der einen Seite steht die Invariabilität eines objektiven Gegenstandes, auf der anderen die Variabilität subjektiver Wahrnehmungen von erfahrbaren Erscheinungen empirisch gegebener Dinge an sich. Daß ein solcher Wahrnehmungsgegenstand selbst variabel ist, ändert nichts an der Tatsache, daß seine Variabilität stets subjektiv wahrgenommen werden muß. Unproblematisch dabei ist, wer da affiziert wird. Es ist das je eigene Affektionssubjekt selbst, das eine von außen her einwirkende Affektion in Form von Sinnesdaten wahrnimmt. Problematisch dagegen ist die Frage, wovon dieses Subjekt affiziert wird. Wie die einschlägige Kant-Rezeption zeigt, entsteht innerhalb der Transzendentalphilosophie das Problem einer adäquaten Affektionserklärung dadurch, daß sich gerade das afficiens und nicht das affectum als äußerst problematisch erweist. Sind es die affizierenden empirischen Dinge in ihrer Vermittlung über ihre Erscheinungen oder irgendwelche transzendenten Dinge-an-sich? Das Affektionsproblem erscheint in der Tat insofern problematisch, als Kant unterschiedliche Sprachausdrücke zur Kennzeichnung eines und desselben Vorgangs gebraucht und dabei keine strikt durchgeführte Begriffspräzisierung vorgenommen hat. Abhilfe kann hier erneut die Sprachanalyse bringen.

Die Annahme einer adäquaten Affektionserklärung im Rahmen folgender Rekonstruktion beruht auf drei Grundprämissen: (1) Kants transzendentaler Ansatz ist konsistent und widerspruchsfrei konzipiert; (2) Die Interpretationsschwierigkeiten resultieren aus einem akuten Mangel an konsequenter Durchführung aufgrund einer semantischen Inkonsequenz in der Verwendungsweise unterschiedlicher Terme zur Kennzeichnung eines und desselben Vorgangs, der die Kantkritiker dazu veranlaßt hat, Kants Lehre für widersprüchlich zu erklären; (3) Die jeweilige Fehlinterpretation beruht auf einem Mißverständnis der Interpreten, die eigene Auffassungsnaivität Kant 
zu unterstellen, und ihn von vornherein auf eine bestimmte Deutungsvariante festzulegen, ohne eine Alternative - die sich als die richtige herausstellen könnte - in Betracht zu ziehen.

Das Problem einer systemimmanenten Affektionserklärung entsteht in bezug auf das Affizierende selbst. Sind es die transzendenten „Dinge-an-sich“ oder lediglich die empirischen Dinge? Beides zugleich kann nicht zutreffen, will man Kant bei der Entwicklung seiner Erfahrungstheorie keinen Widerspruch unterstellen. Wie zuvor gezeigt, reflektiert die zweite Stufe der transzendentalen Betrachtung das nichtempirische Ergebnis der ersten empirischen Stufe in einer Weise, in der sie das transzendentale Resultat über das empirisch Gegebene reflektiert, so wie es sich den Sinnen als Empfindung darbietet. So etwa eine empirisch wahrgenommene Rose als Erscheinung und eine „Rose an sich“, die von einem Subjekt in einer Weise betrachtet werden kann, wie sie ihm jeweils erscheint, ohne das ihre selbsteigene Distinktion dadurch berührt wäre. Daß sie auch „an sich selbst“ betrachtet werden kann, heißt, daß sie auf die transzendentale Reflexionsebene übertragen, in Abhebung von aller Sinnlichkeit als transzendentaler Verstandesgegenstand in seinem Objektsein gedacht und zum Gegenstand im transzendentalen Reflexionssinne überhaupt erst gemacht wird.

Wenn Kant von „Dingen an sich“ spricht - soviel muß vorausgesetzt werden - so meint er im Rahmen seiner Erfahrungstheorie stets empirische Dinge als Erscheinungen, die allein erfahrbar sind, und nicht irgendwelche übersinnlichen „Dinge-an-sich“ jenseits aller empirischen Realität.

Versuchen wir diese Annahme am Beispiel einer „Rose“ zu veranschaulichen. Eine empirisch affizierende Rose als Mannigfaltiges ihrer sinnlichen Anschauung muß, sofern sie als Erscheinungsgegenstand erkannt werden soll, zu einem Vorstellungsganzen - einer Denkeinheit in der transzendentalen Apperzeption des „Ich denke“ - verbunden bzw. synthetisiert werden. Dies geschieht durch einen inneren Grund als Bedingung, die von Kant aber mißverständlicherweise auch als ,intelligible Ursache“ bezeichnet wird. Aus dieser Inkonsequenz haben seine frühen Interpreten den naiven Schluß einer - vermeintlich - transzendenten Kausalverbindung von „Dingen-ansich“ gezogen und daraufhin die gesamte Theorie für inkonsistent erklärt. Dagegen ist einzuwenden, daß wenn eine Kausalverbindung als affizierender Gegenstandsbezug zwischen Subjekt und Objekt als Erscheinung gewahrt bleibt, kann wohl kaum von einer transzendenten Anwendung der Kausalität die Rede sein, die Kant in Verkennung seiner ursprünglichen Absichten vor allem von Jacobi, Schulze und Schopenhauer unterstellt war. 
Die erkenntnistheoretische Verwirrung einer adäquaten Affektionsbestimmung entsteht aus einer semantischen Inkonsequenz, wenn Kant das Begriffspaar Erscheinung und Ding an sich der genuin transzendentalen Reflexionsstufe als Phaenomena und Noumena auftretend, auch als empirische Terme der empirischen Erkenntnisart betrachtet. Wie nachlässig er dabei mit seinen Termini umgeht und die Stufendifferenz nicht einhält, legt folgendes Beispiel nahe: „Wenn ich alle Dinge nicht als Phänomene, sondern als Dinge an sich betrachte, und als Gegenstände des bloßen Verstandes, sie, obschon sie Substanzen sind, dennoch wie abhängig ihrem Dasein nach von fremder Ursache angesehen werden können; welches aber alsdenn ganz andere Wortbedeutungen nach sich ziehen, und auf Erscheinungen, als mögliche Gegenstände der Erfahrung, nicht passen würde“ (KrV B 251/252). Daß durch solche Inkonsequenzen Kant selbst Anlaß zu einer mißverständlichen Affektionsannahme durch „Dinge an sich“ geliefert hat, folgt mutatis mutandis aus ihrer wechselvollen Rezeptionsgeschichte. Das Problem wird nämlich von vornherein einseitig dahingehend thematisiert, als ob Kant zum einen eine kausale Affektion durch transzendente Dinge-an-sich, zum anderen eine Affektion durch empirische Dinge lehrte. Im Anschluß an diese Fehlinterpretation wird die irreführende These einer „Doppelaffektion“ eingeführt ${ }^{13}$.

An dieser Stufenverwirrung setzt Prauss das fragile Affektionsproblem an, denn beide Behauptungen zugleich können nicht zutreffen. Hält man an der Transzendentalphilosophie als widerspruchsfreier Erfahrungstheorie konsequent fest, so dürfte ausgeschlossen sein, den Schluß auf eine transzendente Metaphysik zu ziehen, da diese ja als Erkenntnis der „Dinge an sich“ für Kant ohnehin nicht in Frage kommt. Die Verstandesgrundsätze stellen „bloße Prinzipien der Exposition der Erscheinungen“ dar, so daß „der stolze Name einer Ontologie, welche sich anmaßt, von Dingen überhaupt synthetische Erkenntnisse a priori in einer systematischen Doktrin

${ }^{13}$ Vgl. E. Adickes: Kants Lehre von der doppelten Affektion unseres Ich, Tübingen 1929; ders.: Kant und das Ding an sich, Berlin 1924. Auf dieses Problem hat zunächst Jacobi hingewiesen, bis Adickes schließlich die Annahme einer "doppelten Affektion“ entwickelte. Da Prauss' Analyse aber plausibel nahe legt, daß es in Kants Erfahrungstheorie von einer solchen „doppelten Affektion“ keine Rede sein kann, und Prauss mit der Darstellung der „empirischen Affektion" den richtigen Sinn des Problems rekonstruiert sowie der Annahme einer „Doppelaffektion“ ihre Grundlage entzogen hat, können alle früheren Erklärungsversuche ausgeklammert bleiben. Zur Kritik an dieser Theorie vgl. Prauss, Kant und das Problem der Dinge an sich, S. $193 \mathrm{ff}$. 
zu geben (z. E. den Grundsatz der Kausalität) muß dem bescheidenen, einer bloßen Analytik des reinen Verstandes, Platz machen“ (KrV B 304).

Erst in völliger Verkennung des transzendentalen Ansatzes war es fälschlicherweise möglich, Kant die Einführung transzendent-metaphysischer Affektionsabsichten zu unterstellen. Hält man an einer den Erscheinungscharakter der Erfahrungserkenntnis überschreitenden Kausalaffektion durch ontologisch eigenständige „Dinge-an-sich“ fest, so kommt man in der Tat nicht umhin, Kant eine unmittelbare transzendent-metaphysische Kausalverbindung zu vindizieren, die alsdann das systematische Problem der Dinge an sich überhaupt erst aufwirft. Unbestreitbar allerdings bleibt, daß Kant selbst Anlaß zu dieser Fehlinterpretation gegeben hat. Begreift man seine Philosophie aber als Erfahrungstheorie, und sucht ihre systematische Durchführung anhand von eindeutig formulierten Textbelegen aufzuweisen - die mangels Durchsichtigkeit in der Systematik seiner zweideutigen Ausdrücke unterzugehen scheinen - so entsteht ein völlig anderes Gesamtbild.

Dabei wollen wir Kant mit Kant selbst zur Hilfe kommen, indem wir mitbehaupten, daß es nichts Ungewöhnliches sei, in den Gedanken, die ein Autor über einen Gegenstand geäußert habe, „ihn so gar besser zu verstehen, als er sich selbst verstand, indem er seinen Begriff nicht genugsam bestimmte, und dadurch bisweilen seiner eigenen Absicht entgegen redete, oder auch dachte" (KrV B 370). Zunächst die kontroverse Stelle: „Dasjenige Etwas, welches den äußeren Erscheinungen zum Grunde liegt, was unseren Sinn so affiziert, daß die Vorstellungen von Raum, Materie, Gestalt etc. bekommt, dieses Etwas, als Noumenon (oder besser, als transzendentaler Gegenstand) betrachtet, könnte doch auch zugleich das Subjekt der Gedanken sein, wiewohl wir durch Die Art, wie unser äußere Sinn dadurch affiziert wird, keine Anschauung von Vorstellungen, Willen etc., sondern bloß vom Raum und dessen Bestimmungen bekommen" ( $K r V$ A 358).

Dagegen gibt es eine Vielzahl von klar formulierten Textstellen, die Kants Affektionstheorie in Verbindung mit empirischen Dingen unmißverständlich zum Ausdruck bringen, die von seinen Kritikern aber entweder nicht zur Kenntnis genommen oder gar geleugnet wurden. Erst infolge dieser Mißachtung war es möglich, Kants Theorie für unhaltbar zu erklären. Hält man an der zuvor rekonstruierten zweistufigen Systematik der Transzendentalphilosophie fest, dann erscheint die Sachlage in einem völlig anderen Licht. Denn dieses Etwas ,ist nicht ausgedehnt, nicht undurchdringlich, nicht zusammengesetzt, weil alle diese Prädikate nur die Sinnlichkeit und 
deren Anschauung angehen, sofern wir von dergleichen (uns übrigens unbekannten) Objekten affiziert werden“ $(\mathrm{KrV} \text { A } 358)^{14}$.

Weiter im Text kommt unsere Lesart noch deutlicher zum Tragen: „Diese Ausdrücke aber geben gar nicht zu erkennen, was vor ein Gegenstand es sei, sondern nur: daß ihm, als einem solchen, der ohne Beziehung auf äußere Sinne an sich selbst betrachtet wird, diese Prädikate äußerer Erscheinungen beigelegt werden können“ ( $K r V$ A 358/359). Sinngemäß heißt es auch in der Grundlegung zur Metaphysik der Sitten: „Sobald dieser Unterschied [zwischen Erscheinung und Ding an sich - A. L.] einmal gemacht ist, so folgt von selbst, daß man hinter den Erscheinungen doch noch etwas anderes, was nicht Erscheinung ist, nämlich die Dinge an sich, einräumen und annehmen müsse, ob wir gleich uns von selbst bescheiden, daß, da sie uns niemals bekannt werden können, sondern immer nur, wie sie uns affizieren, wir ihnen nicht näher treten und, was sie an sich sind, niemals wissen können" (GMS, A 106). Damit dürfte hinlänglich geklärt sein, daß Kant stets von einer Affektion durch empirische Dinge ausgegangen ist. Eine erfahrungsrelevante Affektion durch Noumena aufgrund einer transzendent-metaphysischen Kausalverbindung mit hypostasierten „Dingen-an-sich“ kann somit als mit der Transzendentalphilosophie unvereinbar ausgeschlossen werden.

Kants Affektionsaussagen stellen keine transzendenten Kausalbehauptungen unter vermeintlicher Anwendung des Kausalitätsprinzip dar. Auch in diesem Zusammenhang gilt der in die Vernunftkritik programmatisch festgeschriebene Satz: „Wenn aber die Kritik nicht geirrt hat, da sie das Objekt in zweierlei Bedeutung nehmen lehrt, nämlich als Erscheinung, oder als Ding an sich selbst; wenn die Deduktion ihrer Verstandesbegriffe richtig ist, mithin auch der Grundsatz der Kausalität nur auf Dinge im ersten Sinne genommen, nämlich sofern sie Gegenstände der Erfahrung sind“ (KrVXXVII). Die Problemlösung liegt demnach darin, daß man die Annahme einer „Doppelaffektion" verwirft. In diesem Fall gewinnen wir im Ausgang vom Erkenntnissubjekt als Erfahrungsträger eine Doppelbetrachtung, aber keine Doppelaffektion. Es sind eindeutig die empirischen Dinge als Erfahrungsgegenstände, die den Affektionsvorgang einleiten. Wenn Kant dabei semantisch undiffernziert von der Affektion durch „Dinge an sich“ spricht, so ist dies als eine Affektion durch die empirischen Dinge zu verstehen, die auf der

${ }^{14}$ Diesen Sachverhalt stellt Prauss, Kant und das Problem der Dinge an sich, S. 198 f. dar. Zur Affektion durch empirische Dinge vgl. die von Prauss herangezogenen Stellen aus den Prolegomena, Ibid., S. 202 f. 
transzendentalen Betrachtungsebene auch „an sich selbst“ betrachtet werden müssen.

\section{Das transzendentale Objekt als Ding an sich}

Erfahrungsgegenstände sind nach Kant weder „Dinge an sich“ noch assoziativ verbundene Empfindungskonglomerate von zufälligen Vorstellungselementen. Vielmehr stellen sie erfahrbare Objekte dar, die als Mannigfaltiges sinnlicher Anschauungsdaten über die Einheit der transzendentalen Apperzeption unter Vermittlung von Verstandeskategorien zu einer gesetzmäßigen Vorstellungseinheit synthetisiert werden. Erfahrungsobjekte sind sie dem transzendentalen Reflexionsstandpunkt gemäß nur dadurch, daß ein transzendentales Objekt als begrifflicher Korrelationsgedanke auf das Anschauliche bezogen wird. Dieses "transzendentale Objekt“ stellt einen noumenalen Einheitspunkt für subjektiv-psychische Objektivierungsvorgänge des Anschauungsmaterials dar. Es ist das gesuchte Ding an sich $=X$, wie es als Reflexionsgrund - und nicht intelligible Ursache! - der besonderen objektiven Phänomene gedacht wird. Sein empirisches Pendant, die Erscheinung, weist zwei Seiten auf. Die eine, wie Kant selber sagt, „da das Objekt an sich selbst betrachtet wird (unangesehen der Art, dasselbe anzuschauen, dessen Beschaffenheit aber eben darum jederzeit problematisch bleibt)“, die andere dagegen, „da auf die Form der Anschauung dieses Gegenstandes gesehen wird, welche nicht in dem Gegenstande an sich selbst, sondern im Subjekte, dem derselbe erscheint, gesucht werden muß, gleichwohl aber der Erscheinung dieses Gegenstandes wirklich und notwendig zukommt" ( $K r V$ B 56).

Erfahrungstheoretische Erkenntnis war für Kant stets nur unter subjektiven Sinnlichkeits- und Verstandesbedingungen möglich, denn „ohne Sinnlichkeit würde uns kein Gegenstand gegeben, und ohne Verstand keiner gedacht werden“ ( $K r V$ B 75). Diese Reziprozität der Erfahrungserkenntnis - die formal betrachtet aus Anschauungs- und Denkformen besteht - macht es aber möglich, angeschaute Dinge, wie sie den Sinnen erscheinen, und gedachte Dinge, wie sie der Verstand denkt, als „Phänomena“ und „Noumena“ zu betrachten. Ein „Noumenon“ als Gegenstand einer sinnlichen Anschauung bleibt dennoch negativ. Das heißt, der Verstand vermag Dinge nur in einer Form zu erkennen, wie sie unter Raumzeitbedingungen erscheinen, und nicht wie sie „,an sich“ sind: „Und da Erscheinungen nichts als Vorstel- 
lungen sind, so bezieht sie der Verstand auf ein Etwas, als den Gegenstand der sinnlichen Anschauung: aber dieses Etwas ist in sofern nur das transzendentale Objekt. Dieses bedeutet aber ein Etwas = X, wovon wir gar nichts wissen, noch überhaupt [...] wissen können, sondern welches nur als ein Correlatum der Einheit der Apperzeption zur Einheit des Mannigfaltigen in der sinnlichen Anschauung dienen kann, vermittelst deren der Verstand dasselbe in den Begriff eines Gegenstandes vereinigt" ( $K r V$ A 250/251).

Diese Auffassung als legitimen Standpunkt vertrat Kant unverändert auch im Opus postumum. Von einem transzendentalphilosophischen Standpunkt aus nämlich muß die Anschauungsbeziehung eines Gegenstandes unter Raum- und Zeitbedingungen die Unterscheidung machen „zwischen der Vorstellung des Dinges an sich und der eben desselben Dinges aber als Erscheinung, ob wir zwar jenem keine Prädicate beylegen können, sondern es als $=\mathrm{X}$, bloß als Correlatum für den reinen Verstand nicht dabile, sondern nur als cogitabile betrachten, wo die Begriffe nicht die Sachen gegen einander gestellt werden. " Denn , alle Sinnengegenstände sind Dinge in der Erscheinung (obiecta phaenomena), denen ein Noumenon als Grund ihrer Zusammenstellung correspondiert, dem aber keine besondere Anschauung (kein noumenon aspectabile) correspondiert" (Op. post. II, 33). Als Produkt der Denkspontaneität stellt das transzendentale Objekt keine Abstraktion von Anschauungsdaten dar, sondern die noumenale Vorstellung der Erscheinungen unter einem (zunächst) inhaltsleeren Gegenstandsbegriff, der durch das Mannigfaltige der Erscheinungssynthesis erst sukzessiv bestimmbar wird.

Noumenon in negativer Hinsicht heißt, daß die sinnliche Anschauungsart ihre objektive Gültigkeit nicht für alle Dinge hat, sondern auf Gegenstände unter subjektiven Raumzeitbedingungen begrenzt wird. So gesehen ist das Noumenon als Gegenstandsvorstellung ein problematischer Grenzbegriff, der unter empirischen Bedingungen unaufweisbar ist: „Der Begriff des Noumenon ist also nicht der Begriff von einem Objekt, sondern die unvermeidlich mit der Einschränkung unserer Sinnlichkeit zusammenhängende Aufgabe, ob es nicht von jener ihrer Anschauung ganz entbundene Gegenstände geben möge, welche Frage nur unbestimmt beantwortet werden kann, nämlich: daß, weil die sinnliche Anschauung nicht auf alle Dinge ohne Unterschied geht, für mehr und andere Gegenstände Platz übrig bleibe, sie also nicht schlechthin abgeleugnet, in Ermangelung eines bestimmten Begriffs aber (da keine Kategorie dazu tauglich ist) auch nicht als Gegenstände für unsern Verstand behauptet werden können" (KrV B 344). Auf diese Weise schränkt 
der Verstand mit seinem Noumenon die Sinnlichkeit ein, ohne ihren Gegenstandsbereich zu transzendieren.

Die abschließende Lösung der komplexen „Ding an sich“-Frage könnte uns Kants Opus postumum bringen. Während die Vernunftkritik zu folgenschweren Ambiguitäten in bezug auf eine adäquate Interpretation der „Dinge an sich" und ihre verstrickte Verursachungsart geführt und Kant das Problem nicht mehr plausibel auszuräumen vermocht hat, scheint er in den postumen Reflexionen eine adäquate Lösung gefunden zu haben. Denn das „Ding an sich“ wird dem transzendentalen Standpunkt gemäß nicht mehr als ,intelligible Ursache“ gefaßt, sondern als Prinzip, und zwar „das Princip der synthetischen Erkenntnis a priori des Mannigfaltigen der Sinnenanschauung überhaupt" (Op. post. II, 33). Als subjektiver Reflexionsausdruck wird es in seiner Eigenschaft als Grund seiner eigenen Bestimmung gesetzt, der als reines Verstandesobjekt das Formale der Einheit des Mannigfaltigen einer sinnlichen Anschauung enthält.

Eine transzendente Affektion über einen vermeintlichen Kausalschluß, der Kant seitens der Kritiker immer wieder unterstellt war, erweist sich somit als unhaltbar und ist als obsolete Fehldeutung endgültig fallen zu lassen. Das „Ding an sich“ ist weder ein besonderer Erfahrungsgegenstand noch ein seiendes Wesen, sondern ein Prinzip. Als Relationsbegriff wird es nicht außerhalb der Vorstellungsmäßigkeit als reales Ding betrachtet, sondern als abstraktes Gedankending, das in noumenaler Korrelation zum Realen über eine subjektive Setzung als Grund, als Bedingung für die formale Möglichkeit synthetischer Erkenntnis a priori, notwendig gedacht wird (vgl. Op. post. II, 23).

Der Sache nach gehört der Begriff ,intelligible Ursache“, den Kant zuvor mißverständlich eingeführt hat, in den Zusammenhang um den intelligiblen Handlungscharakter des Menschen als Thema der dritten Antinomie der Transzendentalen Dialektik, in der das transzendentale Subjekt als Erscheinung und Ding an sich konstituiert wird, und nicht in den Zusammenhang um das Ding an sich als transzendentales Objekt der Transzendentalen Analytik der Vernunftkritik. Formal kündigt die Unterscheidung in Phaenomena und Noumena die Wendung zum intelligiblen Freiheitscharakter zwar an, sie wird aber nicht in extenso vollzogen. Darin wird abermals die Unterscheidung in Erscheinung und Ding an sich manifest. Denn wären Erscheinungen Dinge an sich, so wäre Freiheit als transzendentale Idee undenkbar und die Naturkausalität die einzige Verursachungsart. Insofern ist Kants unpräziser Ausdrucksweise anzulasten, die in dieser Form einem offenen Durchfüh- 
rungsmangel gleichkommt, daß er diese Unterscheidung im Textlaut der Vernunftkritik nicht strikt ausdifferenziert hat, wenn er in bezug auf das transzendentale Objekt als Ding an sich zur Kennzeichnung ein und desselben Vorgangs abwechselnd von „Grund“ und „Ursache“ spricht.

Abschließend kann resümiert werden, daß das „Ding an sich“ von Kant nicht über transzendente Affektions- oder Kausalvorgänge erschlossen, sondern als besonderes Theoriekonstrukt gesetzt wird. Es ist ein für die Transzendentalphilosophie insgesamt denknotwendiges Prinzip, das über eine subjektive Setzung als problematischer Grenzbegriff eingeführt wird, und dem wegen seiner noumenalen Negativität weder empirische Realität noch metaphysische Distinktion zu vindizieren ist. Indem es als Prinzip designiert wird, schließt es den transzendentalidealistischen Standpunkt insgesamt kohärent ab und braucht nicht länger als „,̈̈bersinnliche Ursache" der Erscheinungen gekennzeichnet werden, die Kant aller Wahrscheinlichkeit nach nur deshalb unterläuft, weil er nachlässig unterschiedliche Terme zur Kennzeichnung ein und desselben Vorgangs gebraucht.

\section{Literatur}

Adickes E., Kant und das Ding an sich, Berlin 1924.

Adickes E., Kants Lehre von der doppelten Affektion unseres Ich, Tübingen 1929.

Kant I., Werkausgabe in 12 Bänden. Hrsg. v. Wilhelm Weischedel. Frankfurt/M. 1974.

Lorenz A., „Problem rzeczy samych w sobie” w filozofii transcendentalnej Kanta - próba rekonstrukcji na podstawie analizy językowej, in: Idea transcendentalizmu. Od Kanta do Wittgensteina, P. Parszutowicz/M. Soin (red.), Warszawa 2011, 51-76.

Lorenz A., Das Problem der Dinge an sich bei Kant, Schopenhauer und Freud, Wrocław 2004.

Mittelstraß J., Ding als Erscheinung und Ding an sich. Zur Kritik einer spekulativen Unterscheidung, in: J. Mittelstraß/M. Riedel (Hg): Vernünftiges Denken. Studien zur praktischen Philosophie und Wissenschaftstheorie, Berlin/New York 1978, 107-123.

Prauss G., Erscheinung bei Kant. Ein Problem der „Kritik der reinen Vernunft“, Berlin 1973.

Prauss G., Kant und das Problem der Dinge an sich, Bonn 1974. 


\begin{abstract}
The Problem of the "Thing-in-Itself" in Immanuel Kant's Philosophy from the Linguistic Analysis Perspective

The paper deals with the problem of the "thing in itself" in Kant's Transcendental Philosophy from the perspective of linguistic analysis. The consideration is conducted with regard to Gerold Prauss's work Kant und das Problem der Dinge an sich. The aim of the paper is to demonstrate the coherence of the Dinge an sich selbst betrachtet concept in its twofold meaning: (1) transcendental-philosophical, that is argumentatively properly justified within the frame of Kant's Theory of Experience, and (2) as a transcendental-metaphysical (non)sense that critics have been focused on since the beginnings of reception of Kant's Philosophy. Such a transcendentmetaphysical (non)sense is understood as an argument in favour of an inconsistency in Kant's thought. Prauss argues, that the non(sense) mentioned above occurs always when Kant - having in mind the methodological distinction of a "thing as a phenomenon" and on the other hand the "thing-in-itself" established within the frame of his own transcendental-philosophical concept - does not consistently observe the implication that results from his own fundamental distinction. It means that Kant in the Critique of Pure Reason had himself contributed to the confusing interpretation of the "thing in itself".
\end{abstract}

Key words: "thing-in-itself", Immanuel Kant, Gerold Prauss, transcendental philosophy 